PROCEEDINGS OF THE

AMERICAN MATHEMATICAL SOCIETY

Volume 126, Number 2, February 1998, Pages 397-404

S 0002-9939(98)04116-1

\title{
ON THE TAYLOR SPECTRUM OF LEFT-RIGHT MULTIPLIERS
}

\author{
ROBIN HARTE AND CARLOS HERNÁNDEZ
}

(Communicated by Palle E. T. Jorgensen)

\begin{abstract}
The Taylor spectrum of a mixed pair of multiplication operators
\end{abstract} is determined on an ultraprime Banach algebra.

Suppose $A$ is a complex Banach algebra, with identity 1 and invertible group $A^{-1}$ (more generally, much of what we have to say may make sense in an additive category $A$ ); then we can define various kinds of "joint spectrum" for systems of elements in $A$ :

Definition 1. Write

$$
\sigma_{A}^{\text {left }}\left(A^{n}\right)=\left\{a \in A^{n}: 1 \notin \sum_{j=1}^{n} A a_{j}\right\} \text { and } \sigma_{A}^{\text {right }}\left(A^{n}\right)=\left\{a \in A^{n}: 1 \notin \sum_{j=1}^{n} a_{j} A\right\},
$$

$$
\pi_{A}^{\text {left }}\left(A^{n}\right)=\left\{a \in A^{n}: L_{a}^{-1}(0) \neq\{0\}\right\} \text { and } \pi_{A}^{\text {right }}\left(A^{n}\right)=\left\{a \in A: R_{a}^{-1}(0) \neq\{0\}\right\}
$$

and

and

$$
\tau_{A}^{\text {left }}\left(A^{n}\right)=\left\{a \in A^{n}: \inf _{\|x\| \geq 1}\left\|L_{a} x\right\|=0\right\}
$$

$$
\tau_{A}^{\text {right }}\left(A^{n}\right)=\left\{a \in A^{n}: \inf _{\|x\| \geq 1}\left\|R_{a} x\right\|=0\right\} ;
$$

then for each $\omega=\sigma^{\text {left }}, \sigma^{\text {right }}, \pi^{\text {left }}, \pi^{\text {right }}, \tau^{\text {left }}, \tau^{\text {right }}$ set for arbitrary $a \in A^{n}$

$$
\omega_{A}(a)=\left\{\lambda \in \mathbb{C}^{n}: a-\lambda \in \omega_{A}\left(A^{n}\right)\right\} .
$$

This slightly eccentric notation reproduces the definitions ([4], Definitions 1.1 1.3) of the left and right spectrum, point and approximate spectrum of an $n$-tuple of Banach algebra elements. We shall also write

$$
\omega_{A}(a)=\omega_{A}^{\text {left }}(a) \cup \omega_{A}^{\text {right }}(a)
$$

for each $\omega=\sigma, \tau, \pi$. We have, for each $a \in A^{n}$ and $x \in A$,

$$
L_{a}(x)=a x \in A^{n} \text { and } R_{a}(x)=x a \in A^{n},
$$

Received by the editors July 16, 1996.

1991 Mathematics Subject Classification. Primary 46L05, 47A10, 47C15, 18G35, 47D25; Secondary 47A62.

Key words and phrases. Taylor spectrum, multiplication operators, prime algebras, ultraprime algebras.

This paper was written while the first named author was visiting the Instituto de Matemáticas, UNAM. 
giving the product $A^{n}$ any one of the obvious norms. Thus

$$
L_{a}^{-1}(0)=\bigcap_{j}\left\{x \in A: a_{j} x=0\right\} \text { and } R_{a}^{-1}(0)=\bigcap_{j}\left\{x \in A: x a_{j}=0\right\}:
$$

in an abus de notation we shall find it convenient to write also

$$
L_{a}\left(A^{n}\right)=\sum_{j} a_{j} A \text { and } R_{a}\left(A^{n}\right)=\sum_{j} A a_{j} .
$$

We recall how these spectra behave under homomorphisms and polynomial mappings:

Lemma 2. If $a \in A^{n}$ and if $p: A^{n} \rightarrow A^{m}$ is a polynomial system then there is inclusion

$$
p \omega_{A}(a) \subseteq \omega_{A} p(a),
$$

with equality for commuting systems of elements when $\omega$ is either spectrum or approximate point spectrum. If $T \in H B L(A, B)$ is a bounded homomorphism between Banach algebras (preserving identity) then there is inclusion

$$
\omega_{B}(T a) \subseteq \omega_{A}(a) \text { for } \omega=\sigma^{\text {left }}, \sigma^{\text {right }} .
$$

If in addition $T$ is one-one there is inclusion

$$
\omega_{A}(a) \subseteq \omega_{B}(T a) \text { for } \omega=\pi^{\text {left }}, \pi^{\text {right }},
$$

and if in addition $T$ is bounded below inclusion

$$
\omega_{A}(a) \subseteq \omega_{B}(T a) \text { for } \omega=\tau^{\text {left }}, \tau^{\text {right }} .
$$

Proof. This is well known ([3], Theorem 3.2; [7], Theorems 11.2.2, 11.3.4, 11.1.4).

In the particular case $B=B L(A, A)$ of the algebra of bounded linear operators on $A$ and either the homomorphism $T: a \mapsto L_{a}$ or the antihomomorphism $T: a \mapsto$ $R_{a}$ of (1.5) we can say more:

Lemma 3. If $a \in A^{n}$ is arbitrary there are equalities

$$
\begin{gathered}
\pi_{A}^{\text {left }}(a)=\pi_{B}^{\text {left }}\left(L_{a}\right) \text { and } \pi_{A}^{\text {right }}(a)=\pi_{B}^{\text {left }}\left(R_{a}\right), \\
\tau_{A}^{\text {left }}(a)=\tau_{B}^{\text {left }}\left(L_{a}\right) \text { and } \tau_{A}^{\text {right }}(a)=\tau_{B}^{\text {left }}\left(R_{a}\right)
\end{gathered}
$$

and

$$
\sigma_{A}^{\text {left }}(a)=\pi_{B}^{\text {right }}\left(R_{a}\right)=\tau_{B}^{\text {right }}\left(R_{a}\right) \text { and } \sigma_{A}^{\text {right }}(a)=\pi_{B}^{\text {right }}\left(L_{a}\right)=\tau_{B}^{\text {right }}\left(L_{a}\right) .
$$

Proof. This is again familiar ([4], Theorem 2.4; [8], Theorem 5.6.3).

From Lemma 2 and Lemma 3 we can make some observations about joint spectra for a mixed system $\left(L_{a}, R_{b}\right) \in B^{n} \times B^{m}$ induced by $(a, b) \in A^{n} \times A^{m}$ :

Theorem 4. If $a \in A^{n}$ and $b \in A^{m}$ is arbitrary then, with $B=B L(A, A)$, there are inclusions

$$
\begin{gathered}
\sigma_{B}^{\text {left }}\left(L_{a}, R_{b}\right) \subseteq \sigma_{A}^{\text {left }}(a) \times \sigma_{B}^{\text {right }}(b) \text { and } \sigma_{B}^{\text {right }}\left(L_{a}, R_{b}\right) \subseteq \sigma_{A}^{\text {right }}(a) \times \sigma_{B}^{\text {left }}(b), \\
\tau_{B}^{\text {left }}\left(L_{a}, R_{b}\right) \subseteq \tau_{A}^{\text {left }}(a) \times \tau_{B}^{\text {right }}(b) \text { and } \tau_{B}^{\text {right }}\left(L_{a}, R_{b}\right) \subseteq \tau_{A}^{\text {right }}(a) \times \tau_{B}^{\text {left }}(b)
\end{gathered}
$$

and

$$
\pi_{B}^{\text {left }}\left(L_{a}, R_{b}\right) \subseteq \pi_{A}^{\text {left }}(a) \times \pi_{B}^{\text {right }}(b) \text { and } \pi_{B}^{\text {right }}\left(L_{a}, R_{b}\right) \subseteq \pi_{A}^{\text {right }}(a) \times \pi_{B}^{\text {left }}(b) .
$$


Proof. This is (2.1) from Lemma 2 together with Lemma 3.

When in particular $A$ itself is an algebra of operators then ([5], Theorem 3.2) we can say more; what we can say extends to algebras $A$ which are "ultraprime":

Definition 5. The Banach algebra $A$ is said to be prime if there is implication, for arbitrary $a, b$ in $A$,

$$
L_{a} R_{b}=0 \Rightarrow 0 \in\{a, b\},
$$

and we shall say that $A$ is ultraprime if there is equality, for arbitrary $a, b$ in $A$,

$$
\left\|L_{a} R_{b}\right\|=\|a\|\|b\| .
$$

We shall call $A$ spectrally prime if there is equality, for arbitrary $a, b$ in $A$,

$$
\sigma_{B}\left(L_{a} R_{b}\right)=\sigma_{A}(a) \sigma_{A}(b) .
$$

It is clear at once that ultraprime algebras are prime; Mathieu ([10], Proposition 2.3) has shown that for $C^{*}$ algebras all three conditions are equivalent.

To see how Lemma 3 improves for ultraprime algebras we introduce two auxiliary spectrum-like objects, writing ( $c f .(3.2 .4)$ and (3.2.5) of [5]) for arbitrary $a \in A^{n}$ and $b \in A^{m}$

$$
\begin{aligned}
& \varpi^{\text {left,right }}\left(A^{n} \times A^{m}\right)= \\
& \quad\left\{(a, b) \in A^{n} \times A^{m}: \inf _{\|x z y\| \geq 1}\|a x|\||| z|||| y||+\| x||||z||| \mid y b\|=0\right\}
\end{aligned}
$$

and

$$
\begin{aligned}
& \varpi^{\text {right,left }}\left(A^{n} \times A^{m}\right)= \\
& \quad\left\{(a, b) \in A^{n} \times A^{m}: \inf _{|| x z y|| \geq 1}\|x a|\||| z|||| y||+\| x|\||| z|||| b y \|=0\right\},
\end{aligned}
$$

and then setting

$$
\omega_{A}(a, b)=\left\{(\lambda, \mu) \in \mathbb{C}^{n} \times \mathbb{C}^{m}:(a-\lambda, b-\mu) \in \omega\left(A^{n} \times A^{m}\right)\right\}
$$

in both cases.

Theorem 6. If $B=B L(A, A)$ there are inclusions, for arbitrary $a \in A^{n}$ and $b \in A^{m}$,

$$
\varpi_{A}^{\text {left,right }}(a, b) \subseteq \tau_{B}^{\text {left }}\left(L_{a}, R_{b}\right)
$$

and

$$
\varpi_{A}^{\text {right,left }}(a, b) \subseteq \tau_{B}^{\text {right }}\left(L_{a}, R_{b}\right) .
$$

If in particular the algebra $A$ is ultraprime then there are also inclusions

$$
\tau_{A}^{\text {left }}(a) \times \tau_{A}^{\text {right }}(b) \subseteq \varpi_{A}^{\text {left,right }}(a, b)
$$

and

$$
\tau_{A}^{\text {right }}(a) \times \tau_{A}^{\text {left }}(b) \subseteq \varpi_{A}^{\text {right,left }}(a, b) .
$$

Proof. This is the argument of Theorem 3.2 of [5] (cf. [8], Theorem 5.6.4): if $(0,0)$ is not in the right-hand side of (6.1) there are $k>0$ and $h>0$ for which, for arbitrary $w \in A$,

$$
\|w\| \leq k\|a w\|+h\|w b\|:
$$


now substitute $x y z$ for $w$. If $(0,0)$ is not in the right-hand side of $(6.2)$ then there are $k>0$ and $h>0$ for which, for arbitrary $z \in A$, there are $a^{\prime} \in A^{n}$ and $b^{\prime} \in A^{m}$ for which

$$
\sum_{j} a_{j} a_{j}^{\prime}+\sum_{i} b_{i}^{\prime} b_{i}=z \text { with }\left\|a^{\prime}\right\| \leq k\|z\| \text { and }\left\|b^{\prime}\right\| \leq h\|z\|:
$$

now multiply on either side by $x$ and $y$. Notice an application of the open mapping theorem here, a point which was missed in the argument of Theorem 3.2.4 of [5].

Conversely if $(0,0)$ is in the left-hand side of $(6.3)$ then there are sequences $\left(x_{r}\right)$ and $\left(y_{r}\right)$ in $A$ for which $\left\|x_{r}\right\|=\left\|y_{r}\right\|=1$ with $\left\|L_{a} x_{r}\right\|+\left\|R_{b} y_{r}\right\| \rightarrow 0$, and then by ultraprimeness $\left(z_{r}\right)$ in $A$ for which $\left\|z_{r}\right\| \leq 2$ with $\left\|x_{r} z_{r} y_{r}\right\|=1$. This puts $(0,0)$ in the right-hand side of (6.3); the proof of (6.4) is similar

If we only assume that $A$ is prime there is an analogue of Theorem 6 in which $\tau$ is replaced by $\pi$ : we leave the reader to write down the analogues of (5.5) and (5.6) that are appropriate. From Theorem 6 we can deduce a spectral mapping theorem for polynomials in $L_{a}$ and $R_{b}$ :

Theorem 7. If $A$ is ultraprime and if $a \in A^{n}$ and $b \in A^{m}$ are arbitrary then for arbitrary systems of polynomials $p: A^{n} \times A^{m} \rightarrow A^{q}$ there are equalities

$$
\tau_{B}^{\text {left }} p\left(L_{a}, R_{b}\right)=p\left(\tau_{A}^{\text {left }}(a), \tau_{A}^{\text {right }}(b)\right) \text { and } \tau_{B}^{\text {right }} p\left(L_{a}, R_{b}\right)=p\left(\tau_{A}^{\text {right }}(a), \tau_{A}^{\text {left }}(b)\right) .
$$

In particular for single elements $a=a_{1} \in A$ and $b=b_{1} \in A$ and for one polynomial $p=p_{1}$ in two variables there is equality

$$
\sigma_{B} p\left(L_{a}, R_{b}\right)=p\left(\sigma_{A}(a), \sigma_{A}(b)\right) .
$$

It follows that there is implication

$$
\text { A ultraprime } \Rightarrow \text { A spectrally prime. }
$$

Proof. The first part is clear from Theorem 6; for the second recall ([5], Theorem 1.3; [8], Theorem 11.6.7) that for compact sets $K$ and $H$ in $\mathbf{C}$ and one polynomial in two variables there is equality

$$
p(\partial K, \partial H)=p(\partial(K \times H))=p(K \times H) .
$$

For the implication (7.3) apply (7.2) with the polynomial $p(z, w)=z w$.

When the algebra $A$ is prime, or ultraprime, then it is of interest to try and determine the "Taylor spectrum" and the "Taylor split spectrum" of systems $\left(L_{a}, R_{b}\right)$ on the Banach space $A$. These are defined through the medium of the Koszul complex:

Definition 8. If $T \in B L(X, X)^{n}$ is a tuple of bounded linear operators on a Banach space $X$ the the Koszul complex of $T$ may be realised as the operator

$$
\Lambda(T): \sum_{k} x_{k} d z_{k} \mapsto \sum_{j=1}^{n} \sum_{k} T_{j} x_{k} d z_{j} \wedge d z_{k}
$$

acting on the tensor product $X \otimes \Lambda(d z)$ of the space $X$ with the exterior algebra on $n$ generators $d z_{j}$; for a true complex

$$
\Lambda(T)^{2}=\mathbf{0}
$$


we need the operators $T_{j}$ to commute with one another. Whether or not this is the case we shall call the system $T$ Taylor non-singular iff ([11]; [6]; [8], Definition 11.9.3)

$$
\Lambda(T)^{-1}(0) \subseteq \Lambda(T) \Lambda(X, d z),
$$

and Taylor invertible ([6]; [8], Definition 11.9.3) iff there are $\mathbf{U}$ and $\mathbf{V}$ on $\Lambda(X, d z)$ for which

$$
\mathbf{V} \Lambda(T)+\Lambda(T) \mathbf{U}=\mathbf{I}
$$

We shall write, with $B=B L(X, X)$,

$$
\operatorname{Sp}_{X}(T)=\tau_{B}^{\text {Taylor }}(T)=\left\{\lambda \in \mathbb{C}^{n}: T-\lambda I \text { is not Taylor nonsingular }\right\}
$$

and

$$
\operatorname{Sp}_{X}^{\text {split }}(T)=\sigma_{B}^{\text {Taylor }}(T)=\left\{\lambda \in \mathbb{C}^{n}: T-\lambda I \text { is not Taylor invertible }\right\} .
$$

Note the inclusion ([11]; [8], Theorem 11.9.9)

$$
\tau_{B}^{\text {Taylor }}(T) \subseteq \sigma_{B}^{\text {Taylor }}(T) \subseteq \prod_{j} \sigma_{B}\left(T_{j}\right) .
$$

Exactness of the Koszul complex of a system of operators induces a lot of exactness between the operators themselves ( $c f$. [9], Theorem 4):

Lemma 9. If $T \in B^{n}$ is Taylor non-singular and $\{k\} \subseteq\{1,2, \ldots, n\}$ then there is inclusion

$$
\bigcap_{j \notin\{k\}} T_{j}^{-1}(0) \subseteq \sum_{j \in\{k\}} T_{j}(X) .
$$

Proof. We are writing $\{k\}=\left\{k_{1}, k_{2}, \ldots, k_{p}\right\}$ if $k=k_{1} k_{2} \ldots k_{p}$ is a multiindex. If $x_{k} \in X$ then by $(8.3)$

$$
\Lambda(T)\left(x_{k} d z_{k}\right)=0 \Rightarrow x_{k} d z_{k} \in \Lambda(T) \Lambda(X, d z)
$$

which means that

$$
\sum_{j \notin\{k\}} T_{j} x_{k} d z_{j k}=0 \Rightarrow x_{k} d z_{j k}=\sum_{j \ell= \pm k} T_{j} x_{\ell} d x_{k} .
$$

Two special cases are familiar: if $\{k\}=\emptyset$ this says that the intersection of the null spaces of the $T_{j}$ is $\{0\}$, while if $\{k\}=\{1,2, \ldots, n\}$ this says that the sum of the ranges of the $T_{j}$ is $X$.

For pairs of operators $T \in B^{2}$ (commuting or not) we can write

$$
\tau_{B}^{\text {Taylor }}(T)=\tau_{B}^{\text {left }}(T) \cup \tau_{B}^{\text {middle }}(T) \cup \tau_{B}^{\text {right }}(T),
$$

where

$$
(0,0) \notin \tau_{B}^{\text {middle }}(T) \Leftrightarrow\left(\Lambda_{1}(T), \Lambda_{0}(T)\right) \text { not exact. }
$$

For a pair of bounded linear operators $U: X \rightarrow Y$ and $V: Y \rightarrow Z$ between Banach spaces (whether or not $V U=0$ ) "exactness" means

$$
V^{-1}(0) \subseteq U(X) \subseteq Y \text { with } V(Y)=\operatorname{cl} V(Y) \subseteq Z:
$$


equivalently ([7], Theorem 2.3; [8], Theorem 10.5.4), there are $k>0$ and $h>0$ for which for arbitrary $y \in Y$ there is $x \in X$ satisfying

$$
\|y-U x\| \leq k\|V y\| \text { and }\|x\| \leq h\|y\| .
$$

Towards a determination of the Taylor spectrum of a pair $\left(L_{a}, R_{b}\right)$ in an ultraprime Banach algebra $A$, we begin with no assumption:

Theorem 10. If $x, y$ are in $A$ and $a \in A^{n}, b \in A^{m}$ there are implications

$$
\begin{aligned}
& x \in L_{a}^{-1}(0), y \in R_{b}^{-1}(0) \Rightarrow\left(L_{x} R_{y}\right)(A) \subseteq L_{a}^{-1}(0) \cap R_{b}^{-1}(0) ; \\
& x \in R_{a}^{-1}(0), y \in L_{b}^{-1}(0) \Rightarrow L_{a}\left(A^{n}\right)+R_{b}\left(A^{m}\right) \subseteq\left(L_{x} R_{y}\right)^{-1}(0) ; \\
& x \in L_{a}^{-1}(0), y \in L_{b}^{-1}(0) \Rightarrow\left(L_{x} R_{y}\right)(A) \subseteq L_{a}^{-1}(0) L_{b}^{-1}(0) ; \\
& x \in R_{a}^{-1}(0), y \in R_{b}^{-1}(0) \Rightarrow\left(L_{x} R_{y}\right)(A) \subseteq R_{a}^{-1}(0) R_{b}^{-1}(0) .
\end{aligned}
$$

Proof. Clear (but remember the notation (1.7) and (1.8)).

When the algebra $A$ is prime then all the point spectrum of $a$ and $b$ is in the spectrum of $\left(L_{a}, R_{b}\right)$ :

Theorem 11. If $A$ is prime then for arbitrary $a \in A^{n}$ and $b \in A^{m}$ there is inclusion

$$
\pi_{A}(a) \times \pi_{A}(b) \subseteq \tau_{B}^{\text {Taylor }}\left(L_{a}, R_{b}\right) .
$$

Proof. To see this break the product $\pi_{A}(a) \times \pi_{A}(b)$ into the union of four products of left and right spectra and then combine each of the four implications of Theorem 10 with the assumption that $A$ is prime. Thus if $(0,0) \in \pi_{A}^{\text {left }}(a) \times \pi_{A}^{\text {right }}(b)$ then there are $x, y, z \in A$ with $a x=y b=0 \neq x z y$, so that by $(10.1) L_{a}^{-1}(0) \cap R_{b}^{-1}(0) \neq\{0\}$. If instead $(0,0) \in \pi_{A}^{\text {right }}(a) \times \pi_{A}^{\text {left }}(b)$ then there are $x, y, z \in A$ with $x a=b y=0 \neq x z y$, so that by $(10.2) L_{a}\left(A^{n}\right)+R_{b}\left(A^{m}\right) \neq A$. If instead $(0,0) \in \pi_{A}^{\text {left }}(a) \times \pi_{A}^{\text {left }}(b)$ then there are $x, y, z \in A$ with $a x=b y=0 \neq x z y$, so that by $(10.3) L_{a}^{-1}(0) L_{b}^{-1}(0) \neq\{0\}$. Since of course $R_{b}(A) L_{b}^{-1}(0)=\{0\}$ this means $L_{a}^{-1}(0) \subseteq R_{b}\left(A^{m}\right)$; now recall (9.1). The argument for the fourth and final case is exactly the same.

We have used here the argument of Curto and Hernández ([3], proof of Theorem 2): notice the inclusions

$$
\sigma_{A}^{\text {left }}(a) \backslash \sigma_{A}^{\text {right }}(a) \subseteq \pi_{A}^{\text {left }}(a) \text { and } \sigma_{A}^{\text {right }}(a) \backslash \sigma_{A}^{\text {left }}(a) \subseteq \pi_{A}^{\text {right }}(a) .
$$

It would be nice if at this point we could pass from the Banach algebra $A$ to its "enlargement"

$$
\mathbf{Q}(A)=\ell_{\infty}(A) / c_{0}(A),
$$

so that the approximate point spectra of $a$ and $b$ would coincide with the point spectrum of their images in the enlargement, while the Taylor spectrum of $\left(L_{a}, R_{b}\right)$ would remain unchanged: we would want the enlargement of an ultraprime algebra to be prime. Unfortunately with this simple-minded concept of enlargement that cannot happen: if $A \neq \mathbf{O}$ then the sequences

$$
a=(1,0,1,0, \ldots), b=(0,1,0,1, \ldots)
$$


satisfy

$$
a \notin c_{0}(A) ; b \notin c_{0}(A) ; a \ell_{\infty}(A) b=\{0\} \subseteq c_{0}(A) .
$$

Thus we need to get our hands dirty, and look at exactness (9.5). When the pair $(V, U)=\left(\left(\begin{array}{cc}-S & T\end{array}\right),\left(\begin{array}{c}T \\ S\end{array}\right)\right)$ forms the Koszul complex of a pair of operators $(S, T)$ on a Banach space $X$ then as in Lemma 9 a necessary condition for exactness is that each of the pairs $(S, T)$ and $(T, S)$ is also exact, this time in the sense of $(9.5)$ :

Theorem 12. If $A$ is ultraprime then for arbitrary $a \in A$ and $b \in A$ there is inclusion

$$
\tau_{A}(a) \times \tau_{A}(b) \subseteq \tau_{B}^{\text {Taylor }}\left(L_{a}, R_{b}\right) \subseteq \sigma_{A}(a) \times \sigma_{A}(b) .
$$

Proof. The second inclusion is (8.7). Towards the first we already know by Theorem 6 that two of the products are included:

$$
\tau_{A}^{\text {left }}(a) \times \tau_{A}^{\text {right }}(b) \subseteq \tau_{B}^{\text {left }}\left(L_{a}, R_{b}\right)
$$

and

$$
\tau_{A}^{\mathrm{right}}(a) \times \tau_{A}^{\mathrm{left}}(b) \subseteq \tau_{B}^{\mathrm{right}}\left(L_{a}, R_{b}\right)
$$

We claim

$$
\tau_{A}^{\mathrm{left}}(a) \times \tau_{A}^{\mathrm{left}}(b) \subseteq \tau_{B}^{\text {middle }}\left(L_{a}, R_{b}\right)
$$

and

$$
\tau_{A}^{\text {right }}(a) \times \tau_{A}^{\text {right }}(b) \subseteq \tau_{B}^{\text {middle }}\left(L_{a}, R_{b}\right) .
$$

Thus suppose for a contradiction that $(0,0)$ is in the left-hand side of (12.4) but not in the right: thus there are $\left(x_{r}\right)$ and $\left(y_{r}\right)$ in $A$ for which

$$
\left\|x_{r}\right\|=\left\|y_{r}\right\|=1 \text { with }\left\|a x_{r}\right\|+\left\|b y_{r}\right\| \rightarrow 0 .
$$

Now if $(0,0)$ is not in the right-hand side of (12.4) then both $\left(R_{b}, L_{a}\right)$ and $\left(L_{a}, R_{b}\right)$ are exact in the sense of (9.5). Taking the first alternative there is, for arbitrary $z \in A, w_{r} \in A$ for which

$$
\left\|x_{r} z-w_{r} b\right\| \leq k\left\|a x_{r} z\right\| \text { with }\left\|w_{r}\right\| \leq h\left\|x_{r} z\right\|:
$$

it follows

$$
\begin{gathered}
\left\|x_{r} z y_{r}\right\| \leq\left\|x_{r} z-w_{r} b\left|\left\|\mid y_{r}\right\|+\left\|w_{r}\right\|\left\|b y_{r}\right\| \leq\right.\right. \\
k\left\|a x _ { r } \left|\left\|| | z \left|\left\|y_{r}\right\|+h\left\|x_{r}\right\|\left\|z \left|\left\||| b y_{r}\right\| \rightarrow 0 .\right.\right.\right.\right.\right.\right.
\end{gathered}
$$

By uniform boundedness ([8], Theorem 4.9.1) it follows that

$$
\sup _{\|z\| \leq 1}\left\|x_{r} z y_{r}\right\| \rightarrow 0
$$

and then by ultraprimeness $\left\|y_{r}\right\|\left\|x_{r}\right\| \rightarrow 0$, a contradiction. If instead $(0,0)$ is in the left-hand side of (12.5) then apply (9.5) with $L_{a}$ and $R_{b}$ reversed.

If either $A=B L(X, X)$ or $A$ is a $C^{*}$-algebra, so that there is equality

$$
\sigma_{A}(a)=\tau_{A}(a) \text { for each } a \in A,
$$

then of course [3] there is equality throughout (12.1). An extension of the first inclusion of (12.1) to $a \in A^{n}$ and $b \in A^{m}$ would follow from the analogue of Lemma 9 with the exactness condition (9.5). 


\section{REFERENCES}

1. R.E. Curto, Applications of several complex variables to multiparameter spectral theory, Pitman lecture notes 1985. MR 90d:47007

2. R.E. Curto and L.A. Fialkow, The spectral picture of $\left(L_{a}, R_{b}\right)$, Jour. Funct. Anal. 71 (1987) 371-392. MR 88c:47006

3. R.E. Curto and C. Hernández G., A joint spectral characterization of primeness for $C^{*}$ algebras, Proc. Amer. Math. Soc. (to appear). CMP 96:16

4. R.E. Harte, Spectral mapping theorems, Proc. Royal Irish Acad. 72A (1972) 89-107. MR 48:4738

5. R.E. Harte, Tensor products, multiplication operators and spectral mapping theorems, Proc. Royal Irish Acad. 73A (1973) 285-302. MR 48:6984

6. R.E. Harte, Invertibility, singularity and Joseph L. Taylor, Proc. Royal Irish Acad. 81A (1981) 71-79. MR 82m:47003

7. R.E. Harte, Almost exactness in normed linear spaces, Proc. Amer. Math. Soc. 100 (1987) 257-265. MR 88g:47004

8. R.E. Harte, Invertibility and singularity (Dekker New York 1988).

9. R.E. Harte, Taylor exactness and Kato's jump, Proc. Amer. Math. Soc. 119 (1993) 793-801. MR 93m:47020

10. M. Mathieu, Elementary operators on prime $C^{*}$-algebras I, Math. Ann. 284 (1989) 223-244. MR 90h:46092

11. J.L. Taylor, A joint spectrum for several commuting operators, Jour. Funct. Anal. 6 (1970) 172-191. MR 42:3603

School of Mathematics, Trinity College, Dublin 2, Ireland

E-mail address: rharte@maths.tcd.ie

Instituto de Matemáticas, UNAM, Ciudad Universitaria, México, D.F. 04510, México

E-mail address: carlosh@matem.unam.mx 